\title{
02.2
}

\section{Резонансная перезарядка в электростатическом поле}

\author{
(C) А.З. Девдариани ${ }^{1,2}$, А.О. Артамонова ${ }^{1}$, А.К. Беляев ${ }^{2}$ \\ ${ }^{1}$ Санкт-Петербургский государственный университет, Санкт-Петербург, Россия \\ ${ }^{2}$ Российский государственный педагогический университет им. А.И. Герцена, Санкт-Петербург, Россия \\ E-mail: snbrn2@yandex.ru
}

Поступило в Редакцию 18 июля 2019г.

В окончательной редакции 18 ноября 2019г.

Принято к публикации 22 ноября 2019 г.

\begin{abstract}
Проведена оценка влияния электростатического поля, существующего, например, в плазме, на процесс резонансной перезарядки при скоростях столкновений, существенно меньших скорости перезарядочного электрона, в рамках модели потенциалов малого радиуса. Установлено, что наложение поля достаточной величины приводит к уменьшению сечения перезарядки. Например, в случае реакции $\mathrm{H}^{-}+\mathrm{H} \rightarrow \mathrm{H}+\mathrm{H}^{-}$ поле $\sim 10^{7} \mathrm{~V} / \mathrm{cm}$ уменьшает сечение перезарядки на порядок.
\end{abstract}

Ключевые слова: плазма, резонансная перезарядка, электрическое поле, сечение, потенциал малого радиуса.

DOI: 10.21883/PJTF.2020.04.49051.17990

Свойства плазмы в значительной степени определяются процессом перезарядки, однако обычно, например при расчете транспортных свойств, используются формулы для сечения перезарядки, которые не учитывают наличия поля, хотя оно всегда присутствует в плазме (см., например, [1]. Цель настоящей работы - на примере интересной для астрофизики и физики горячей плазмы реакции $\mathrm{H}^{-}+\mathrm{H} \rightarrow \mathrm{H}+\mathrm{H}^{-}$оценить влияние электростатического поля на процесс резонансной перезарядки при скоростях столкновений, существенно меньших скорости перезарядочного электрона.

Для описания взаимодействия дополнительного электрона с нейтральными атомами используем модель потенциалов малого радиуса, которая оправдана для процессов с участием ионов $\mathrm{H}^{-}$[2]. Поскольку влияние поля наиболее существенно для столкновений, ось которых совпадает с направлением поля, достаточно рассмотреть одномерный случай. Тогда потенциал взаимодействия электрона с атомами в поле напряженности $F$ записывается как

$$
U=-\alpha \delta\left(x-\frac{R}{2}\right)-\alpha \delta\left(x+\frac{R}{2}\right)+F x,
$$

где первые два члена описывают взаимодействие электрона с атомами водорода, $R$-межатомное расстояние, $\alpha=\sqrt{2|\varepsilon|}, \varepsilon-$ энергия связи электрона в ионе, для иона $\mathrm{H}^{-} \quad \alpha=0.23$ (здесь и далее, если не оговорено другое, все величины приведены в атомных единицах). Отметим, что модель электрона в поле двух потенциалов малого радиуса применяется, например, для описания туннельной ионизации электрона в электрическом поле [3].

Решение уравнения Шредингера

$$
i\left(\frac{\partial}{\partial t} \Psi\right)=H^{e l} \Psi
$$

с электронным гамильтонианом вида

$$
H^{e l}=-\frac{1}{2} \frac{d^{2}}{d x^{2}}+U
$$

представим как

$$
\Psi=c_{r}(t) \varphi_{r}\left(x-\frac{R}{2}\right) e^{-i E_{r} t}+c_{l}(t) \varphi_{l}\left(x+\frac{R}{2}\right) e^{-i E_{l} t},
$$

где $\varphi_{r, l}$ и $c_{r, l}$ - волновые функции и амплитуды вероятности нахождения соответственно у правого и левого атомных центров.

С точностью до слагаемых второго порядка малости по интегралу перекрытия волновых функций амплитуды удовлетворяют следующей системе уравнений:

$$
\left\{\begin{array}{l}
i\left(\frac{\partial}{\partial t} c_{r}(t)\right)=\frac{1}{2} F R c_{r}(t)-\alpha A(R) c_{l}(t), \\
i\left(\frac{\partial}{\partial t} c_{l}(t)\right)=-\alpha A(R) c_{r}(t)-\frac{1}{2} F R c_{l}(t),
\end{array}\right.
$$

где

$$
A(R)=\varphi_{l}^{*}(0) \varphi_{r}(R)=\varphi_{r}^{*}(0) \varphi_{l}(R)=\alpha e^{-\alpha R} .
$$

Как видно из (5), при отсутствии поля процесс описывается традиционными уравнениями для резонансной перезарядки [4]. Взаимодействие с полем нарушает резонанс и приводит к уменьшению сечения перезарядки. Формально в терминах двухуровневых моделей теории атомных столкновений [4] система уравнений (5) может быть описана как модель Ландау-Зинера, но не с постоянным, как принято в этой модели, взаимодействием, а со взаимодействием, которое зависит экспоненциально от межатомного расстояния (рис. 1).

Грубая оценка межатомного расстояния $R_{*}$, при котором происходит нарушение резонанса, следует из 


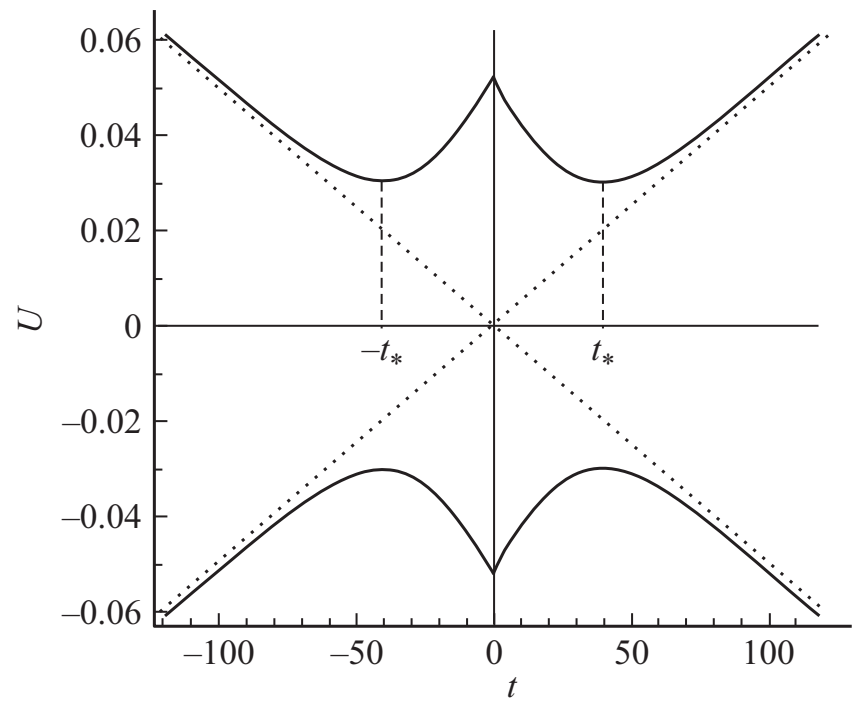

Рис. 1. Схематическая картина адиабатических термов (сплошные линии), обменного взаимодействия и энергии электрона в электрическом поле (пунктир) для реакции перезарядки $\mathrm{H}^{-}+\mathrm{H}$ в поле напряженности $F=1.1 \cdot 10^{-2}$ при скорости атомов $v=9.3 \cdot 10^{-2}, R_{*}=v t_{*}$. Все величины здесь и на остальных рисунках приведены в атомных единицах.

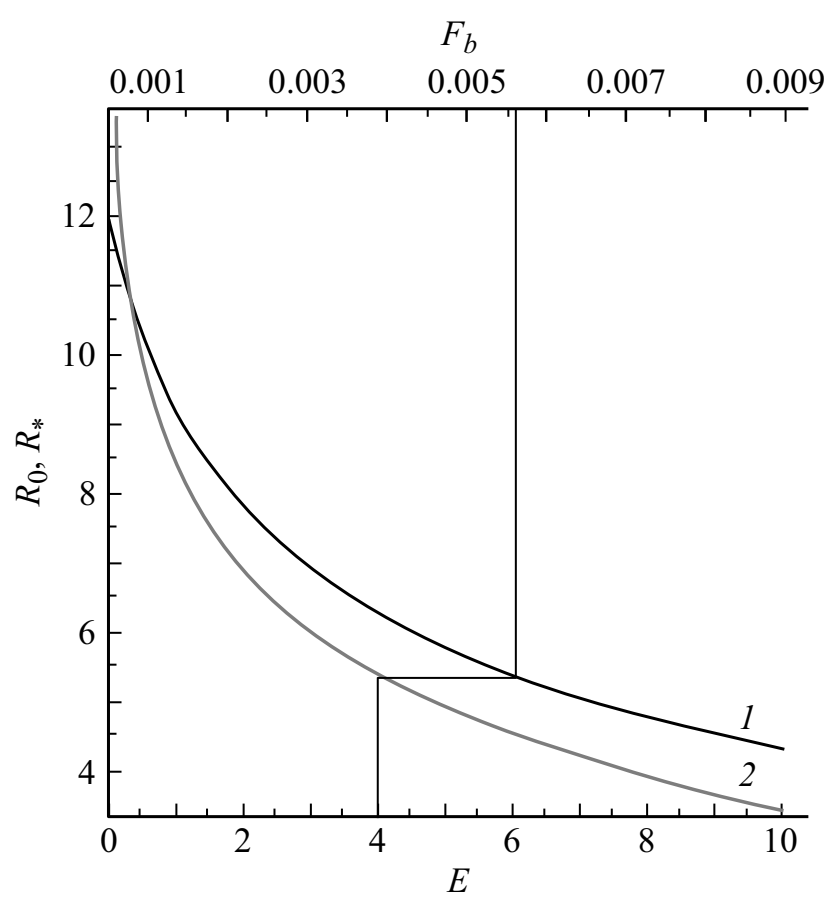

Рис. 2. Зависимость от напряженности расстояния $R_{*}$ между атомами электрического поля (формула (7)) (кривая 1) и зависимость от энергии столкновения $E$ расстояния $R_{0}$ между атомами, при котором вероятность перезарядки $\sim 0.5$ (формула (10)) (кривая 2).

равенства энергии обменного взаимодействия и энергии, приобретаемой (или теряемой) электроном при перехо- де между атомными центрами во внешнем поле:

$$
\alpha A\left(R_{*}\right) \leqslant \frac{1}{2} F R_{*}
$$

или

$$
\alpha^{2} e^{-\alpha R_{*}} \leqslant \frac{1}{2} F R_{*}
$$

Таким образом, при $R>R_{*}$ взаимодействие с полем блокирует резонансный переход электрона между атомными центрами, а при $R<R_{*}$ обменное взаимодействие оказывается преобладающим, так что качественно перезарядка в этой области расстояний происходит обычным образом (рис. 1). Отметим, что условия (7), (8) определяются только свойствами атомов, образующих квазимолекулу, и не зависят от энергии столкновений.

Для численной оценки влияния электрического поля на вероятность перезарядки воспользуемся известным приемом Фирсова [5] для определения границы области расстояний $R_{0}$, внутри которой вероятность перезарядки будет больше $1 / 2$, а именно

$$
\frac{\alpha}{v} e^{-\alpha R_{0}} \sim 0.28
$$

или

$$
R_{0}=-\frac{1}{\alpha} \ln \left(\frac{v}{\alpha}\right)=-\frac{1}{\alpha} \ln \left(\sqrt{\frac{2 E}{M}} \frac{1}{\alpha}\right) .
$$

где $E=M v^{2} / 2$ - энергия налетающей частицы.

Далее строим по формулам (8), (10) зависимости $R_{*}(F), R_{0}(E)$, которые и позволяют проследить влияние электрического поля (рис. 2). Если внешнее поле таково, что для данной энергии столкновения $R_{*}(F)>R_{0}$, то влиянием поля можно пренебречь, так что сечение перезарядки равно $\sigma_{e x}=\pi R_{0}^{2} / 2$ (сплошные горизонтальные

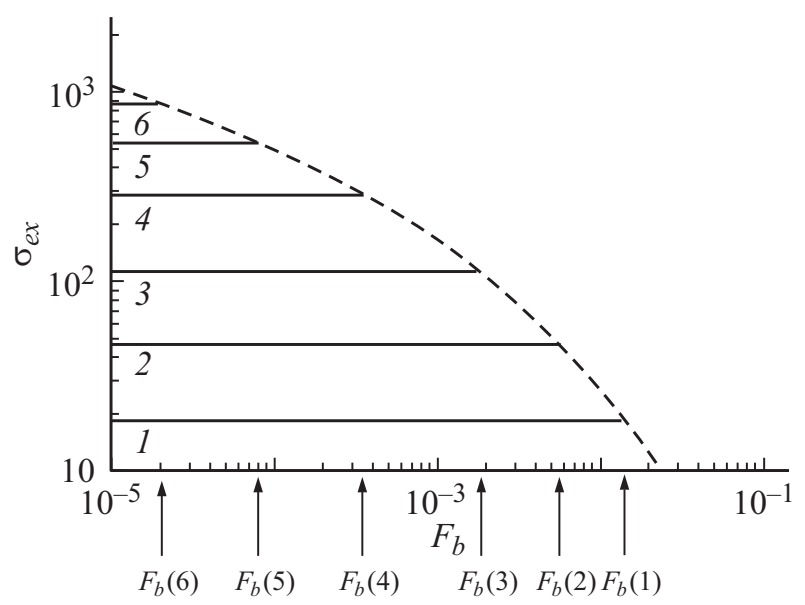

Рис. 3. Зависимость сечения перезарядки от напряженности электрического поля при энергиях столкновений $E(1)=10, E(2)=4, E(3)=1, E(4)=0.1, E(5)=0.01$, $E(6)=0.001$. Минимальное значение поля $F_{b}(1)=1.39 \cdot 10^{-2}$, $F_{b}(2)=5.59 \cdot 10^{-3}, \quad F_{b}(3)=1.79 \cdot 10^{-3}, \quad F_{b}(4)=3.56 \cdot 10^{-4}$, $F_{b}(5)=8.22 \cdot 10^{-5}, F_{b}(6)=2.05 \cdot 10^{-5}$. При $F<F_{b}$ сечение $\sigma_{e x}=\pi R_{0}^{2} / 2$ (сплошные горизонтальные линии 1-6), при $F>F_{b} \sigma_{e x}=\pi R_{*}^{2} / 2$ (штриховая линия). 
линии 1-6 на рис. 3). В частности, это справедливо для предела $F \rightarrow 0$. В противоположном случае, когда $R_{*}(F)<R_{0}$, сечение перезарядки равно $\sigma_{e x}=\pi R_{*}^{2} / 2$ (штриховая кривая на рис. 3, которая не зависит от энергии столкновения).

Для нахождения минимального значения поля $F_{b}$, начиная с которого происходит уменьшение сечения перезарядки, необходимо для данной энергии столкновения определить значение $R_{0}$ по кривой 2 на рис. 2 , а затем по зависимости $R_{*}(F)$ (кривая 1 на рис. 2) определить значение минимального поля $F_{b}$. Построенная таким образом зависимость сечения перезарядки от напряженности поля для различных энергий столкновения приведена на рис. 3. Например, для энергии налетающей частицы, равной 4 , величина $R_{0}(E)=5.4$, так что нижняя граница внешнего электрического поля, которое приводит к уменьшению сечения перезарядки, равна $F_{b}=2.9 \cdot 10^{7} \mathrm{~V} / \mathrm{cm}$.

Как известно, наложение поля приводит к подбарьерной ионизации [6], но оценка вероятности такого процесса

$$
\omega=\tau_{c} \frac{4}{F} e^{-2 / 3 F}
$$

(где $\omega-$ вероятность ионизации в единицу времени, $\tau_{c}$ - время столкновения) показывает, что для полей порядка $F_{b}$ этим процессом можно пренебречь.

Таким образом, подход, основанный на системе уравнений (5), позволяет оценить величину электрического поля, начиная с которого величина сечения резонансной перезарядки становится меньше вычисляемой в рамках традиционного подхода. Несмотря на то что в качестве конкретного процесса рассматривалась перезарядка отрицательных атомарных ионов, для которых использование потенциала малого радиуса вполне оправдано, эффект уменьшения сечения с возрастанием величины внешнего поля будет иметь место и при перезарядке положительных ионов. Возможность моделирования процессов в кулоновском поле с помощью модели потенциала малого радиуса обсуждается в [7].

\section{Благодарности}

Авторы благодарны С.В. Бобашеву за полезное обсуждение, рецензенту за предложение представить графически зависимость сечения от величины поля (рис. 3), Я.В. Воронову за помощь в подготовке рис. 3 .

\section{Финансирование работы}

Работа поддержана Министерством науки и высшего образования РФ (проекты № 3.5042.2017/6.7, 3.1738.2017/4.6).

\section{Конфликт интересов}

Авторы заявляют, что у них нет конфликта интересов.

\section{Список литературы}

[1] Wang H., Sukhomlinov V.S., Kaganovich I.D., Mustafaev A.S. // Plasma Sources Sci. Technol. 2017. V. 26. P. 024001.

[2] Демков Ю.Н., Островский В.Н. Метод потенциалов нулевого радиуса в атомной физике. Л.: Изд-во ЛГУ, 1975. 240 c. [Demkov Yu.N., Ostrovsky V.N. Zero-range potentials and their application in atomic physics. N.Y.: Plenum Press, 1988. 288 p.].

[3] Головинский П.А., Дробышев А.А. // Письма в ЖТФ. 2017. T. 43. В. 4. C. $102-110$.

[4] Галицкий B.М., Никитин Е.Е., Смирнов Б.М. Теория столкновений атомных частиц. М.: Наука, 1981. 256 с.

[5] Смирнов Б.М. // УФН. 2008. Т. 178. № 3. С. 309-311.

[6] Ландау Л.Д., Лифшии Е.М. Квантовая механика. Нерелятивистская теория. М.: Физматлит, 2008. 800 c. [Landau L.D., Lifshitz E.M. Quantum mechanics. Nonrelativistic theory. Oxford: Pergamon Press, 1958. 515 p.].

[7] Sala S., Förster J., Saenz A. // Phys. Rev. A. 2017. V. 95. P. $011403(\mathrm{R})$. 